\title{
Should frames of reference be enacted in astronomy instruction?
}

\author{
Emmanuel Rollinde『* \\ CY Cergy Paris Université, Université de Paris, Univ Paris Est Creteil, Univ. Lille, \\ UNIROUEN, LDAR, F-95000 Cergy-Pontoise, France \\ Nicolas Decamp ${ }^{\circ}$ \\ Université de Paris, Univ Paris Est Creteil, CY Cergy Paris Université, Univ. Lille, \\ UNIROUEN, LDAR, F-75013 Paris, France \\ Catherine Derniaux \\ Lycée Condorcet, 94210 Saint-Maur des Fossés, France
}

(Received 18 April 2020; accepted 21 April 2021; published 7 June 2021)

\begin{abstract}
The experiment that we present in this paper explores the teaching of Galilean motion principles observed in different reference frames, in an astronomical context. All grade 10 students in a French high school (the lycée Condorcet, Val de Marne) participated in two successive teaching-learning sessions, designed within the theoretical framework of embodied cognition. The learning material consisted of two versions of a spatiotemporal aspect map of the Solar System that allowed students to enact and observe trajectories from different points of view. The first was a printed, paper model (PO) that was used individually on a table. The other was a human version (HO). Thus, students enacted movements with either their fingers (PO) or their bodies (HO). Both sessions (HO or PO) used the same activities to illustrate the movements of Earth, Mars, and the Sun during a 24-h and 1-yr period, observed from different reference frames (terrestrial, geocentric, or heliocentric). Students' conceptual understanding was tested using a questionnaire, which was administrated before and after each session, and three months later. The questionnaire described three situations in which the motion of an object is observed from two different points of view. We expected students to understand that speed and distance traveled were different in both cases. Our initial results suggest that the sessions did have a significant and lasting effect on students' understanding of the dependence of motions on reference frames. While the degree of embodiment (HO or PO) does not seem to affect conceptual learning, the abstract operation of moving from one reference frame to another is facilitated when one has physically and repeatedly lived it.
\end{abstract}

DOI: 10.1103/PhysRevPhysEducRes.17.013105

\section{INTRODUCTION}

Recent years have seen a huge increase in the use of space and spatial representations (maps) [1]. This requires specific skills in spatial thinking, which is intrinsically cross disciplinary and therefore not fostered in disciplinecentered educational systems. The specific question of changing perspective-moving from one reference frame to another-is, however, of interest for both physics and mathematics. The key point is the student's ability to think about physical quantities related to movements independent of their definition of a reference frame [2]. This ability is connected to what we call here the relativity principle.

\footnotetext{
*emmanuel.rollinde@cyu.fr
}

Published by the American Physical Society under the terms of the Creative Commons Attribution 4.0 International license. Further distribution of this work must maintain attribution to the author(s) and the published article's title, journal citation, and DOI.
The speed of an object, the distance it travels during a movement, and the shape of its trajectory are a function of the chosen reference frame. This principle is difficult for most people to understand, whatever their age or level of education in classical mechanics [3-6].

Astronomy is a special case where spatial thinking is required to "create accurate mental models of complex phenomena that are too vast to see" $[7,8]$. This complexity, due to a large extent to the absence of a "natural" reference frame [9-13], is suited to the creation of situations that challenge students' conceptions of a natural reference frame, with either Earth moving around the Sun or the Sun around Earth while both are correct [11]. There is a "relative lack of experimental (interventional) studies" that challenge those conceptions [7]. This paper presents an embodied learning approach to address students' difficulties in changing perspective when moving between different reference frames. 


\section{RATIONALE}

\section{A. Embodied learning}

Enaction theory [14] recognizes the role of perception and action in grounding abstract knowledge, and blurs the traditional separation between sensorimotor processes and abstract thought. Typically, the working hypothesis is that by engaging the learner's body in physical enactment (embodied learning), abstract or remote notions are reframed as "tangible" and proximal. This makes the latter more accessible, and facilitates the emergence of refined knowledge structures [15-17].

When learners attempt to make connections between reference frames, they go beyond verbal explanations and call upon physical resources and bodily movements to support their reasoning [18-20]. Sensorial experiences and scientific concepts must be reconnected, particularly through "mindful attention to perception" [14,21,22]. With this objective in mind, we introduce the human Orrery.

\section{B. Spatiotemporal aspect maps of the Solar System}

A planetarium (a dome-shaped projection of the sky) is often used to show the motion of planets around the Sun $[23,24]$. Orreries, mechanical models of the Solar System, are an alternative. Often considered engaging, both are complex and may not be any more efficient for educational purposes than static representations [25]. The latter provide, at zero cognitive cost, all of the "perceptual" inferences, essentially "using vision to think" [26-30]. Hence, hybrid visual displays are considered [31]. They represent planets as visual spatial entities (iconic display), and show their movements (relational display) through the simultaneous representation of all of their positions at different times within a single static image. Drawn in the heliocentric frame, they accurately figure elliptical orbits consistent with Kepler's laws. When presented on an A4 sheet of paper or on a human scale (where $1 \mathrm{~m}$ represents the average Earth-Sun distance), they are known as printed or human Orrery (PO or $\mathrm{HO}$ ). A PO allows the user to observe all planetary trajectories at a glance, and they can trace the movements of the planets around the Sun with their fingers. A HO involves students directly in the experience. Their own actions are central to the demonstration, as they physically enact the movements of the planets (see Sec. I in the Supplemental Material [32]).

\section{A human or printed Orrery}

In the following, we describe aspect maps, $\mathrm{HO}$ and $\mathrm{PO}$ with a specific attention to how the different aspects of those maps are enacted or not.

Entities refers to planets, but no information about physical structures is represented. The spatial scale is shown on the map. Positions in the heliocentric frame at a constant time interval (16 terrestrial days) are shown through a succession of points (each planet is shown in a different color). The evolution of these positions over time may be used to infer relative positions and distance traveled.

Students (HO) or fingers (PO) move along the planetary orbit at a rhythm determined by another person (usually the teacher) who claps his hands regularly. Between two claps, all planets move, simultaneously, from one point to the next, while the Sun stays at rest (in the heliocentric frame). Through these rules (or metaknowledge), users can infer the displacement of any reference frame on the map and, hence, the positions and velocities of any planet in the three astronomical frames (geocentric, heliocentric, and terrestrial). Students extract trajectories enacted in the $\mathrm{HO}$ or $\mathrm{PO}$ in the heliocentric frame to develop a new aspect map that is drawn in another reference frame. This activity helps them to perceive such a mental extraction that will be required to think about change of reference frames.

Frames are enacted in the $\mathrm{HO}$ by the body of the student. Arms represent axes, and one shoulder is the center (see Fig. S3 in Sec. I in the Supplemental Material [32]). By moving their own body, students have a physical perception of speed, while the local trajectory is inferred visually at each position and reproduced on a new map (in an additional sheet of paper). In the PO, frames are enacted as additional sheets of tracing paper (translucent) on which a cross is drawn with axes that are parallel to the sides of the sheet of paper. The tracing paper can be moved and rotated, if required, along the trajectory of the center of the frame (Earth or the Sun), while the positions of other bodies can be plotted as they move. Although the new trajectory is visual, the speed cannot be perceived physically, and has to be inferred through the abstract rules of kinematics.

\section{Research questions}

We developed a series of activities that used a $\mathrm{HO}$ or $\mathrm{PO}$ to extract a new aspect map while moving from the heliocentric frame to the geocentric or terrestrial frame; and to infer general rules about two quantities, namely, distance traveled and speed.

Our first research question was does such an activity, with a focus on perception, promote an understanding of the consequences of the relativity principle on the description of physical quantities in different reference frames? Our methodology used both a $\mathrm{HO}$ and a $\mathrm{PO}$, which only differ with respect to the level of embodiment, as described above. Using the taxonomy given in Ref. [33], the PO is at the second level (interactivity with a learning environment with materials being present on the table) while the HO is at the fourth level (a learning environment that features a high degree of bodily movement involving locomotion). Hence, our second question was: is the level of understanding a function of the level of embodiment? 


\section{METHODOLOGY}

A total of 246 grade 10 students participated (average age 15; see Sec. II in the Supplemental Material [32] for details about French curricula). The sample was randomly split into 16 groups of about 15 students. All groups participated in two sequences: one with a $\mathrm{PO}(\mathrm{P})$ on an $\mathrm{A} 4$ format paper and one with a $\mathrm{HO}(\mathrm{H})$. Eight groups (the $\mathrm{HP}$ set) first participated in the $\mathrm{HO}$ and then the $\mathrm{PO}$. The remaining eight groups $(\mathrm{PH})$ do the reverse $(\mathrm{P}$ and then $\mathrm{H})$. Both sequences lasted about $45 \mathrm{~min}$.

\section{A. Description of the sequence}

The sequences that were used are in line with the principles of enacted task design [34] (p. 153): (i) at least two contrasting examples (here, trajectories) are considered; (ii) students are asked to comment on what is the same or different in the contrasting examples; (iii) the language and notation provided by students are used. Students engagement was coherent with the phenomenological approach presented in Ref. [35], which proposes three phases to "become aware: an initial phase of suspension of habitual thought and judgment, followed by a phase of conversion of attention from "the exterior" to "the interior," ending with a phase of letting-go or receptivity towards the experience." The sequence is described in detail in Sec. II in the Supplemental Material [32].

- In our sequence, the initial phase consists of the discovery of the Orrery: learning to move in it, and a presentation of the different reference frames as explained above.

- Two sets of movements involving Mars, Earth, and the Sun with a constant duration ( $24 \mathrm{~h}$ or $1 \mathrm{yr})$ and three perspectives (heliocentric, terrestrial, or geocentric) are studied and students are asked to plot the trajectories they observe. These observations are, of course, very different depending on the reference frame (see examples of drawings in Sec. 2 of Ref. [32]).

- Once all students have drawn both trajectories within one set, the class is asked to compare what they have obtained: notably, that the speed and distance traveled change from one reference frame to the other, while the duration is the same. Speed is the most discussed notion. Movements may then be repeated (for HO) or drawings can be observed (for both) until all students agree that the speed was not the same.

\section{B. Data collection}

\section{Instrument}

A questionnaire was developed to investigate the evolution of students' knowledge in three contexts: a parachutist who drops his or her goggles (linear movement); circular movement of Jupiter; and a circular movement on a merry go round. For each context, students were asked to answer two questions, whether or not (YES or NO) the distance traveled or the speed of the observed object (the
TABLE I. Chronology of activities in human Orrery and printed Orrery and questionnaires. Activities were led by the first author (1), the second author (2) or a school teacher (ST).

\begin{tabular}{llccccc}
\hline \hline & & & & & & \multicolumn{2}{c}{$\begin{array}{c}\text { Three } \\
\text { months } \\
\text { after }\end{array}$} \\
\hline 8 HP groups & Q1 & HO (1) & Q2 & PO (ST) & Q3 & Q4 \\
8 PH groups & Q1 & PO (2) & Q2 & HO (ST) & Q3 & Q4 \\
\hline \hline
\end{tabular}

goggles, Jupiter or the child on the merry-go-round) was the same for two observers. The correct answer is always NO. We did not ask whether the duration of the movement was the same as duration is not an issue (at least in classical mechanics). These questions and contexts are described in Sec. III in the Supplemental Material [32] and follow the seminal work of Ref. [3].

\section{Procedure}

The experimentation was run between February and May 2019. The same 30-min questionnaire was completed four times. Answers to the questionnaire were never provided to the students. In February 2019, the HP group completed the pre-test questionnaire (Q1), then spent $1 \mathrm{~h}$ in the $\mathrm{HO}$, then completed the post-test1 questionnaire (Q2). Three days later, they engaged in $1 \mathrm{~h}$ of activities with the PO and completed the post-test2 questionnaire (Q3). About three months later, at the end of May 2019, they completed the delayed test questionnaire (Q4). The PH group followed the same procedure, except that they first engaged with the PO and then the HO three days later. For both HP and PH sets, the initial sessions with the $\mathrm{HO}$ or PO were conducted by the first and second authors, respectively. They were observed by teachers who conducted the second sessions with the PO (HP group) or HO (PH group). The methodology is summarized in Table I.

\section{Data analysis}

The comparison of Q1, Q2, and Q3 (McNemar's test) evaluated the immediate efficiency of the sequence on students' conceptions of reference frames and the relativity principle. Q4 evaluated the delayed retention of learning. The comparison of evolution between Q1 and Q2 for the PH and HP sets (Fisher's test) determined the impact of the level of embodiment.

\section{RESULTS AND DISCUSSION}

A total of $228(\mathrm{HP}=117+\mathrm{PH}=111)$ students attended the first session and completed the questionnaires (Q1 and Q2). $222(103+99)$ students attended the second session and answered questionnaire Q3. Lastly, 133 $(73+60)$ students completed the delayed questionnaire (Q4). Numbers are lower than 246 due to student absences. Mean performance as a function of group and assessment is 
TABLE II. The percentage of correct answers to the two questions in the three contexts are shown for each questionnaire (Q1, Q2, Q3, Q4) for HP and PH groups. The total number of students is given in each case.

\begin{tabular}{|c|c|c|c|c|c|c|c|c|c|}
\hline \multirow[b]{2}{*}{ Context } & \multirow[b]{2}{*}{ Question } & \multicolumn{2}{|c|}{ Pretest, Q1 } & \multicolumn{2}{|c|}{ Post-test, Q2 } & \multicolumn{2}{|c|}{ Post-test, Q3 } & \multicolumn{2}{|c|}{ Delayed test, Q4 } \\
\hline & & $\mathrm{HP}$ & $\mathrm{PH}$ & HP & $\mathrm{PH}$ & $\mathrm{HP}$ & $\mathrm{PH}$ & $\mathrm{HP}$ & $\mathrm{PH}$ \\
\hline$N=$ & & 117 & 111 & 117 & 111 & 103 & 99 & 73 & 60 \\
\hline a. Skydivers & & $37 \%$ & $37 \%$ & $44 \%$ & $43 \%$ & $52 \%$ & $53 \%$ & $44 \%$ & $47 \%$ \\
\hline c. Jupiter & Distance & $61 \%$ & $57 \%$ & $84 \%$ & $77 \%$ & $90 \%$ & $74 \%$ & $77 \%$ & $82 \%$ \\
\hline e. Merry go round & & $56 \%$ & $54 \%$ & $72 \%$ & $70 \%$ & $76 \%$ & $71 \%$ & $78 \%$ & $75 \%$ \\
\hline b. Skydivers & & $49 \%$ & $47 \%$ & $55 \%$ & $54 \%$ & $72 \%$ & $67 \%$ & $59 \%$ & $53 \%$ \\
\hline d. Jupiter & Speed & $46 \%$ & $48 \%$ & $68 \%$ & $68 \%$ & $78 \%$ & $71 \%$ & $66 \%$ & $60 \%$ \\
\hline f. Merry go round & & $47 \%$ & $47 \%$ & $62 \%$ & $64 \%$ & $77 \%$ & $68 \%$ & $66 \%$ & $57 \%$ \\
\hline
\end{tabular}

given in Table II. A detailed breakdown of the data and the statistical tests is given in Sec. IV in the Supplemental Material [32] (Tables S-I and S-II).

The results of Q1 are consistent with the hypothesis that the two sets of groups (PH and HP) were initially homogeneous: the percentages of correct answers (to any question), about 50\%, did not differ significantly (see Table II).

\section{A. RQ1: Impact on the level of understanding}

Considering the overall dataset, the percentage of correct answers increased after the first session (Q2, just over 60\%) and again at the end of the second session (70\% in Q3). A significant positive effect was observed for each individual question after the two sessions (column Q1 $\rightarrow$ Q3, Table S-I [32]). The first session alone (column Q1 $\rightarrow$ Q2) generated a significant increase in correct answers to questions related to Jupiter and the merry go round [(c), (d), (e), (f)], but not questions related to the skydivers [(a) and (b)]. Session 2 alone (column Q2 $\rightarrow$ Q3) had no significant effect on distance-related questions, but improved understanding of speed-related questions (except question d). Hence, the change in students' thinking about the nonpreservation of speed seems to occur gradually (over the two sessions), whereas it occurs more rapidly regarding the nonpreservation of distance traveled. If the students were only using the conclusion reached after each activity, the percentage of correct answers should increase similarly for all questions, which is not the case. We hypothesize that the observed evolution reflects rather a progressively enhanced ability to apply the relativity principle.

In response to our first research question, the sessions did have an impact on students' performance. Beyond an overall improvement, the percentage of correct answers depended on the concepts and the context. Proximity with the astronomical context or perceived motion may explain part of these differences: notably, (c) to (f) (rotation) versus (a),(b) (translation). The terrestrial frame, which is particularly present in (a),(b), outweighs the ability to mentally place oneself in the reference frame linked to the skydivers.
With respect to concepts, distance traveled and speed are not treated equally. Observations of the length of the trajectory are visual and immediate in the Orrery (heliocentric) frame. This may have impeded the ability to accept the nonpreservation of distance traveled, like the usual, preferred terrestrial frame in mental reasoning. Nevertheless, our procedure alleviated this obstacle after one session only. The latter finding can be explained by the fact that the non-preservation of speed is perceived directly. Further improvements after the second session may be explained by a deeper understanding of the relation between the nonpreservation of speed, the preservation of duration and the nonpreservation of distance traveled.

\section{B. RQ2: Influence of the degree of embodiment}

During the first session, $\mathrm{HP}$ and $\mathrm{PH}$ groups engaged in the $\mathrm{HO}$ and the PO, respectively (Table S-II [32]). No significant difference was observed in the percentage of correct answers to any of the questions in Q2, or in changes in responses between Q1 and Q2. No difference was noted either between Q1 and Q3 after both groups had engaged in both orreries. The exception was the question about distance in the astronomy context; here engaging in the $\mathrm{HO}$ before the PO improved student's performance, more than the reverse.

We conclude that there is no significant difference concerning the degree of embodiment of $\mathrm{HO}$ versus PO. Our initial prediction was that the higher level of embodiment in the $\mathrm{HO}$ would have a greater effect on our performance measure. In the PO scenario, bodily activities can be considered as incidental. Yet, a high level of embodiment may create a cognitive overload [16]. In our context, the HO provides students with a direct perception of concepts that change, specifically speed. However, in this case, the extraction of the new map has a high cognitive load, compared to almost no cognitive load in the PO setting, where only paper is manipulated. Another issue is that students in the $\mathrm{HO}$ are not always active, while they are continually engaged in the drawing in the $\mathrm{PO}$ (or at least moving the paper). Finally, it should be noted that space in the PO can be perceived from a single vantage 
point while students have to move around in the HO, which creates additional cognitive difficulties [29]. These differences seem to counterbalance each other, especially if we assume that the slight difference observed for only one question is an artifact.

The literature on embodied learning [16,36] highlights that bodily engagement does not automatically improve learning performance. To the best of our knowledge, only one study has shown that embodied conditions lead to better one-week retention in the context of centripetal forces [37]. In our study, we did not have a nonembodied control group. For both embodied conditions, the improvement in understanding remained significant even after three months, except for one question (see Table II and Sec. IV in the Supplemental Material [32]).

\section{CONCLUSION AND PERSPECTIVES}

We propose a specific strategy that can enhance students' understanding of the consequences of the relativity principle on the description of physical quantities in different reference frames, using two versions of a spatiotemporal aspect map of the Solar System, requiring different degrees of embodiment. We conclude that our pedagogical proposition contributes to weaken the students' conception of a natural reference frame whatever the degree of embodiment. We make the hypothesis that students need to have lived experience in their "toolbox" in order to be able to proceed into abstract cognitive activities. Hence, repeatedly perceiving (through the whole body or with the hands) the impact of changing frames of reference facilitates subsequent mental extractions even several months later.

Three main limitations should be noted. We could not implement a true "business-as-usual" control group, as teachers wanted all of their students to participate in the activity; we focus on quantitative results from a large amount of data; the experiment was run by the researchers and not the teachers. Hence, directions for future research are considered now.

First, the transferability of the sequence is not obvious. As the teacher must pay attention to the body of the students, they may feel uncomfortable. In our experiment, teachers took over from researchers in the second session for each group. The rate of correct answers increased whether teachers or researchers supervised the session. Although this appears to be a positive result, conditions were different since students had already participated in the first session before the teachers took over. We plan to reproduce our experiment by delegating the entire management of sessions to teachers for some students, as this could confirm and extend our findings. Second, the next phase of our research will investigate a qualitative analysis of co-speech gestures and specific visuokinetic signs [38] produced by participants to assess their degree of understanding of concepts [39]. Furthermore, we will explore their narrative, introspective, accounts in individual interviews that will be designed following the explicitation methodology [40].
[1] D. G. Janelle, M. Hegarty, and N. S. Newcombe, Spatial thinking across the college curriculum: A report on a specialist meeting, Spatial Cognit. Computation 14, 124 (2014).

[2] S. Joshua, S. Musgrave, N. Hatfield, and P. W. Thompson, Conceptualizing and reasoning with frames of reference, in Proceedings of the 18th Meeting of the MAA Special Interest Group on Research in Undergraduate Mathematics Education, Pittsburgh, Pennsylvania, edited by $\mathrm{T}$. Fukawa-Connelly, N. Infante, K. Keene, and M. Zandieh (2015), pp. 31-44.

[3] E. Saltiel and J. Malgrange, "Spontaneous" ways of reasoning in elementary kinematics, Eur. J. Phys. 1, 73 (1980).

[4] C. W. Camp, J. Clement, D. Brown, K. Gonzalez, J. Kudukey, J. Minstrell, K. Schultz, M. Steinberg, V. Veneman, and A. Zietsman, Preconceptions in Mechanics: Lessons Dealing with Students' Conceptual Difficulties (Kendall/ Hunt, Dubuque, Iowa, 1994).

[5] J. Ramadas, S. Barve, and A. Kumar, Alternative conceptions in galilean relativity: distance, time, energy and laws, Int. J. Sci. Educ. 18, 463 (1996).
[6] L. Viennot, Reasoning in Physics: The Part of Common Sense (Springer Science \& Business Media, New York, 2001).

[7] M. Cole, C. Cohen, J. Wilhelm, and R. Lindell, Spatial thinking in astronomy education research, Phys. Rev. Phys. Educ. Res. 14, 010139 (2018).

[8] S. Vosniadou, Conceptual development in astronomy, in The Psychology of Learning Science, edited by S.M. Glynn, R. H. Yeany, and B. K. Britton (Lawrence Erlbaum Assoc., Hillsdale, NJ, 1991), p. 149.

[9] A. Albanese, M. D. Neves, and M. Vicentini, Models in science and in education: A critical review of research on students' ideas about the earth and its place in the universe, Sci. Educ. 6, 573 (1997).

[10] C. on the Support for the Thinking Spatially, C. on Geography, N. R. Council et al., Learning to Think Spatially: GIS as a Support System in the K-12 Curriculum (National Academies Press, Washington, DC, 2006).

[11] J. Shen and J. Confrey, Justifying alternative models in learning astronomy: A study of K-8 science teachers' understanding of frames of reference, Int. J. Sci. Educ. 32, 1 (2010). 
[12] J. D. Plummer, K. D. Wasko, and C. Slagle, Children learning to explain daily celestial motion: Understanding astronomy across moving frames of reference, Int. J. Sci. Educ. 33, 1963 (2011).

[13] P. Chastenay, From geocentrism to allocentrism: Teaching the phases of the moon in a digital full-dome planetarium, Res. Sci. Educ. 46, 43 (2016).

[14] F. J. Varela, E. Thompson, and E. Rosch, The Embodied Mind: Cognitive Science and Human Experience (MIT Press, Cambridge, MA, 1991).

[15] M. C. Johnson-Glenberg and C. Megowan-Romanowicz, Embodied science and mixed reality: How gesture and motion capture affect physics education, Cogn. Res. Principles Implications 2, 24 (2017).

[16] A. Skulmowski and G. D. Rey, Embodied learning: introducing a taxonomy based on bodily engagement and task integration, Cogn. Res. Principles Implications 3, 6 (2018).

[17] T. G. Amin and O. Levrini, Converging Perspectives on Conceptual Change (Routledge, New York, 2018).

[18] E. M. Crowder, Gestures at work in sense-making science talk, J. Learn. Sci. 5, 173 (1996).

[19] O. Parnafes, Developing explanations and developing understanding: Students explain the phases of the moon using visual representations, Cognit. Instr. 30, 359 (2012).

[20] J. D. Plummer, C. A. Bower, and L. S. Liben, The role of perspective taking in how children connect reference frames when explaining astronomical phenomena, Int. J. Sci. Educ. 38, 345 (2016).

[21] M. Wilson, Six views of embodied cognition, Psychon. Bull. Rev. 9, 625 (2002).

[22] A. M. Glenberg, J. K. Witt, and J. Metcalfe, From the revolution to embodiment: 25 years of cognitive psychology, Perspect. Psychol. Sci. 8, 573 (2013).

[23] B. D. Brazell and S. Espinoza, Meta-analysis of planetarium efficacy research, Astron. Educ. Rev. 8 (2009).

[24] J. D. Plummer, S. Schmoll, K. C. Yu, and C. Ghent, A guide to conducting educational research in the planetarium, Planetarian 44, 8 (2015), http://www.juliaplummer .com/papers/PlummerPlanetariumResearch2015.pdf.

[25] B. Tversky, J. B. Morrison, and M. Betrancourt, Animation: can it facilitate?, Int. J. Human-Comput. Stud. 57, 247 (2002).

[26] J.H. Larkin and H. A. Simon, Why a diagram is (sometimes) worth ten thousand words, Cogn. Sci. 11, 65 (1987).

[27] M. Scaife and Y. Rogers, External cognition: How do graphical representations work?, Int. J. Human-Comput. Stud. 45, 185 (1996).
[28] M. Card, Readings in Information Visualization: Using Vision to Think (Morgan Kaufmann, Burlington, MA, 1999).

[29] B. Berendt, T. Barkowsky, C. Freksa, and S. Kelter, Spatial representation with aspect maps, in Spatial Cognition (Springer, New York, 1998), pp. 313-336.

[30] J. Ramadas, Visual and spatial modes in science learning, Int. J. Sci. Educ. 31, 301 (2009).

[31] M. Hegarty, The cognitive science of visual-spatial displays: Implications for design, Topics Cogn. Sci. 3, 446 (2011).

[32] See Supplemental Material at http://link.aps.org/ supplemental/10.1103/PhysRevPhysEducRes.17.013105

for a full description of the sequence and detailed quantitative results.

[33] M. C. Johnson-Glenberg, D. A. Birchfield, L. Tolentino, and T. Koziupa, Collaborative embodied learning in mixed reality motion-capture environments: Two science studies, J. Educ. Psychol. 106, 86 (2014).

[34] A. Coles and L. Brown, Task design for ways of working: Making distinctions in teaching and learning mathematics, J. Math. Teacher Educ. 19, 149 (2016).

[35] N. Depraz, F. Varela, and P. Vermersch, The gesture of awareness: An account of its structural dynamics, in Investigating Phenomenal Consciousness: New Methodologies and Maps (John Benjamins Publising Co., Amsterdam, 2000), Chap. 6, p. 121-137.

[36] D. DeSutter and M. Stieff, Designing for spatial thinking in stem: Embodying perspective shifts does not lead to improvements in the imagined operations, in Proceedings of the 14th International Conference of the Learning Sciences (ICLS) 2020 (International Society of the Learning Sciences, 2020), Vol. 2, pp. 975-982.

[37] M. C. Johnson-Glenberg, C. Megowan-Romanowicz, D. A. Birchfield, and C. Savio-Ramos, Effects of embodied learning and digital platform on the retention of physics content: Centripetal force, Frontiers Psychol. 7, 1819 (2016).

[38] I. Mittelberg, Visuokinetic signs are inherently metonymic: How embodied metonymy motivates forms, functions, and schematic patterns in gesture, Frontiers Psychol. 10, 254 (2019).

[39] D. McNeill, Gesture and Thought (University of Chicago Press, Chicago, IL, 2008).

[40] P. Vermersch, Describing the practice of introspection, J. Consciousness Studies 16, 20 (2009), https://www.grex2 .com/assets/files/2011Vermersch\%20describing\% 20introspection.pdf. 